\title{
Suzuki-type fixed point theorem for fuzzy mappings in ordered metric spaces
}

Basit $\mathrm{Ali}^{1 *}$ and $\mathrm{MAbbas}{ }^{1,2}$

"Correspondence: basit.aa@gmail.com

'Department of Mathematics, School of Science and Engineering, Lahore University of Management Sciences, Lahore, 54792, Pakistan

Full list of author information is available at the end of the article

\begin{abstract}
In this paper, a Suzuki-type fixed fuzzy point result for fuzzy mappings in complete ordered metric spaces is obtained. As an application, we establish the existence of coincidence fuzzy points and common fixed fuzzy points for a hybrid pair of a single-valued self-mapping and a fuzzy mapping. An example is also provided to support the main result presented herein.
\end{abstract}

MSC: 47H10;47H04;47H07

Keywords: fixed fuzzy point; fuzzy mapping; fuzzy set; approximate quantity

\section{Introduction and preliminaries}

Let $X$ be a space of points with generic elements of $X$ denoted by $x$ and $I=[0,1]$. A fuzzy subset of $X$ is characterized by a membership function such that each element in $X$ is associated with a real number in the interval $I$. Let $(X, d)$ be a metric space and a fuzzy set $A$ in $X$ is characterized by a membership function $A$. Then $\alpha$-level set of $A$, denoted by $A_{\alpha}$, is defined as

$$
A_{\alpha}=\{x: A(x) \geq \alpha\}
$$

for $\alpha \in(0,1]$ and for $\alpha=0$, we have

$$
A_{0}=\overline{\{x: A(x)>0\}},
$$

where $\bar{B}$ denotes the closure of the non-fuzzy set $B$. A fuzzy set $A$ in $X$ is said to be an approximate quantity if and only if for $\alpha \in[0,1], A_{\alpha}$ is a compact, convex subset of $X$ and

$$
\sup _{x \in X} A(x)=1
$$

Let $W(X)$ be a family of all approximate quantities in $X$. A fuzzy set $A$ is said to be more accurate than a fuzzy set $B$ denoted by $A \subset B$ (that is, $B$ includes $A$ ) if and only if $A(x) \leq$ $B(x)$ for each $x$ in $X$, where $A(x)$ and $B(x)$ denote the membership function of $A$ and $B$, respectively. It is easy to see that if $0<\alpha \leq \beta \leq 1$, then $A_{\alpha} \subseteq A_{\beta}$.

(c) 2013 Ali and Abbas; licensee Springer. This is an Open Access article distributed under the terms of the Creative Commons Attribution License (http://creativecommons.org/licenses/by/2.0), which permits unrestricted use, distribution, and reproduction in any medium, provided the original work is properly cited. 
Corresponding to each $\alpha \in[0,1]$ and $x \in X$, the fuzzy point $x_{\alpha}$ of $X$ is the fuzzy set $x_{\alpha}: X \rightarrow[0,1]$ given by

$$
x_{\alpha}(y)= \begin{cases}\alpha & \text { if } x=y, \\ 0 & \text { otherwise }\end{cases}
$$

For $\alpha=1$, we have

$$
x_{1}(y)=\left\{\begin{array}{ll}
1 & \text { if } x=y, \\
0 & \text { otherwise }
\end{array}=\{x\}\right.
$$

Let $I^{X}$ be a collection of all fuzzy subsets of $X$ and $W(X)$ be a subcollection of all approximate quantities. For $A, B \in W(X)$ and $\alpha \in[0,1]$, define

$$
\begin{aligned}
& p_{\alpha}(A, B)=\inf \left\{d(x, y), x \in A_{\alpha}, y \in B_{\alpha}\right\}, \\
& D_{\alpha}(A, B)=\max \left\{\sup _{x \in A_{\alpha}} d\left(x, B_{\alpha}\right), \sup _{y \in B_{\alpha}} d\left(y, A_{\alpha}\right)\right\}
\end{aligned}
$$

and

$$
D(A, B)=\sup _{\alpha} D_{\alpha}(A, B) .
$$

Note that $p_{\alpha}$ is a nondecreasing function of $\alpha$ and $D$ is a metric on $W(X)$. Let $\alpha \in[0,1]$. Define $W_{\alpha}(X)=\left\{A \in I^{X}: A_{\alpha}\right.$ is nonempty, convex and compact $\}$. Let $(X, d)$ be a metric space and $Y$ be an arbitrary set. A mapping $F: Y \rightarrow W_{\alpha}(X)$ is called a fuzzy mapping, that is, $F y \in W_{\alpha}(X)$ for each $y$ in $Y$. Thus, if we characterize a fuzzy set $F y$ in a metric space $X$ by a membership function $F y$, then $F y(x)$ is the grade of membership of $x$ in $F y$. Therefore, a fuzzy mapping $F$ is a fuzzy subset of $Y \times X$ with a membership function $F y(x)$.

In a more general sense than that given in [1], a mapping $F: X \rightarrow I^{X}$ is a fuzzy mapping over $X[2]$ and $(F(x) x)$ is the fixed degree of $x$ in $F(x)$.

Definition 1 ([3]) A fuzzy point $x_{\alpha}$ in $X$ is called a fixed fuzzy point of the fuzzy mapping $F$ if $x_{\alpha} \subset F x$, that is, $(F x) x \geq \alpha$ or $x \in(F x)_{\alpha}$. That is, the fixed degree of $x$ in $F x$ is at least $\alpha$. If $\{x\} \subset F x$, then $x$ is a fixed point of a fuzzy mapping $F$.

Let $F: X \rightarrow W_{\alpha}(X)$ and $g: X \rightarrow X$.

A fuzzy point $x_{\alpha}$ in $X$ is called a coincidence fuzzy point of the hybrid pair $\{F, g\}$ if $(g x)_{\alpha} \subset F x$, that is, $(F x) g x \geq \alpha$ or $g x \in(F x)_{\alpha}$. That is, the fixed degree of $g x$ in $F x$ is at least $\alpha$. A fuzzy point $x_{\alpha}$ in $X$ is called a common fixed fuzzy point of the hybrid pair $\{F, g\}$ if $x_{\alpha}=(g x)_{\alpha} \subset F x$, that is, $x=g x \in(F x)_{\alpha}$ (the fixed degree of $x$ and $g x$ in $F x$ is the same and is at least $\alpha$ ).

We denote by $C_{\alpha}(F, g)$ and $F_{\alpha}(F, g)$ the set of all coincidence fuzzy points and the set of all common fixed fuzzy points of the hybrid pair $\{F, g\}$, respectively.

A hybrid pair $\{F, g\}$ is called $w$-fuzzy compatible if $g(F x)_{\alpha} \subseteq(F g x)_{\alpha}$ whenever $x \in C_{\alpha}(F, g)$. A mapping $g$ is called $F$-fuzzy weakly commuting at some point $x \in X$ if $g^{2}(x) \in(F g x)_{\alpha}$. 
Lemma 1 ([4]) Let $X$ be a nonempty set and $g: X \rightarrow X$. Then there exists a subset $E \subseteq X$ such that $g(E)=g(X)$ and $g: E \rightarrow X$ is one-to-one.

Definition 2 Let $X$ be a nonempty set. Then $(X, d, \preceq)$ is called an ordered metric space if $(X, d)$ is a metric space and $(X, \preceq)$ is partially ordered.

Let $(X, \preceq)$ be a partially ordered set. Then $x, y \in X$ are said to be comparable if $x \preceq y$ or $y \preceq x$ holds.

Define

$$
\nabla=\{(x, y) \in X \times X: x \preceq y \text { or } y \preceq x\} .
$$

An ordered metric space is said to satisfy the order sequential limit property if $\left(u_{n}, z\right) \in \nabla$ for all $n$, whenever a sequence $u_{n} \rightarrow z$ and $\left(u_{n}, u_{n+1}\right) \in \nabla$ for all $n$.

A mapping $F: X \rightarrow W_{\alpha}(X)$ is said to be an ordered fuzzy mapping if the following conditions are satisfied:

(a) $y \in F(x)_{\alpha}$ implies that $(y, x) \in \nabla$.

(b) $(x, y) \in \nabla$ implies that $(u, v) \in \nabla$ whenever $u \in(F x)_{\alpha}$ and $v \in(F y)_{\alpha}$.

The following lemmas are needed in the sequel.

Lemma 2 (Heilpern [1]) Let $(X, d)$ be a metric space, $x, y \in X$ and $A, B \in W(X)$ :

1. if $p_{\alpha}(x, A)=0$, then $x_{\alpha} \subset A$;

2. $p_{\alpha}(x, A) \leq d(x, y)+p_{\alpha}(y, A)$;

3. if $x_{\alpha} \subset A$, then $p_{\alpha}(x, B) \leq D_{\alpha}(A, B)$.

Lemma 3 (Lee and Cho [5]) Let $(X, d)$ be a complete metric space and $F$ be a fuzzy mapping from $X$ into $W(X)$ and $x_{0} \in X$. Then there exists an $x_{1} \in X$ such that $\left\{x_{1}\right\} \subset F x_{0}$.

Zadeh [6] introduced the concept of a fuzzy set. Heilpern [1] introduced the concept of fuzzy mappings in a metric space and proved a fixed point theorem for fuzzy contraction mappings as a generalization of the fixed point theorem for multivalued mappings given by Nadler [7]. Estruch and Vidal [3] proved a fixed point theorem for fuzzy contraction mappings in complete metric spaces which in turn generalizes the Heilpern fixed point theorem. Further generalizations of the result given in [3] were proved in [8,9]. Recently, Suzuki [10] generalized the Banach contraction principle and characterized the metric completeness property of an underlying space. Among many generalizations (see [11-13]) of the results given in [10], Dorić and Lazović [14] obtained Suzuki-type fixed point results for a generalized multivalued contraction in complete metric spaces.

On the other hand, the existence of fixed points in ordered metric spaces has been introduced and applied by Ran and Reurings [15]. Fixed point theorems in partially ordered metric spaces are hybrid of two fundamental principles: Banach contraction theorem with a contractive condition for comparable elements and a selection of an initial point to generate a monotone sequence. For results concerning fixed points and common fixed points in partially ordered metrics spaces, we refer to [16-22].

The aim of this paper is to investigate Suzuki-type fixed point results for fuzzy mappings in complete ordered metric spaces. As an application, a coincidence fuzzy point and a 
common fixed fuzzy point of the hybrid pair of a single-valued self-mapping and a fuzzy mapping are obtained. We provide an example to support the result.

Throughout this paper, let $\sigma:[0,1) \rightarrow(0,1]$ be the nonincreasing function defined by

$$
\sigma(r)= \begin{cases}1 & \text { if } 0 \leq r<\frac{1}{2} \\ 1-r & \text { if } \frac{1}{2} \leq r<1\end{cases}
$$

\section{Main results}

The following theorem is the main result of the paper and is a generalization of [14, Theorem 2.1] for fuzzy mappings in ordered metric spaces.

Theorem 4 Let $(X, d, \preceq)$ be a complete ordered metric space. If an ordered fuzzy mapping $F: X \rightarrow W_{\alpha}(X)$ satisfies

$$
\sigma(r) p_{\alpha}(x, F x) \leq d(x, y) \quad \text { implies } \quad D_{\alpha}(F x, F y) \leq r M_{\alpha}(F)
$$

for all $(x, y) \in \nabla$, where

$$
M_{\alpha}(F)=\max \left\{d(x, y), p_{\alpha}(x, F x), p_{\alpha}(y, F y), \frac{p_{\alpha}(x, F y)+p_{\alpha}(y, F x)}{2}\right\} .
$$

Then there exists a point $x \in X$ such that $x_{\alpha} \subset$ Fx provided that $X$ satisfies the order sequential limit property.

Proof Let $r_{1}$ be a real number such that $0 \leq r<r_{1}<1$ and $u_{1} \in X$. Since $\left(F u_{1}\right)_{\alpha}$ is nonempty and compact, there exists $u_{2} \in\left(F u_{1}\right)_{\alpha}$ such that

$$
d\left(u_{1}, u_{2}\right)=p_{\alpha}\left(u_{1}, F u_{1}\right)
$$

By the given assumption, we have $\left(u_{1}, u_{2}\right) \in \nabla$. Since $\left(F u_{2}\right)_{\alpha}$ is nonempty and compact, there exists $u_{3} \in\left(F u_{2}\right)_{\alpha}$ such that

$$
d\left(u_{2}, u_{3}\right)=p_{\alpha}\left(u_{2}, F u_{2}\right) \leq D_{\alpha}\left(F u_{1}, F u_{2}\right) .
$$

Also, $\left(u_{2}, u_{3}\right) \in \nabla$. Since $\sigma(r)<1$, we obtain

$$
\sigma(r) \rho_{\alpha}\left(u_{1}, F u_{1}\right) \leq p_{\alpha}\left(u_{1}, F u_{1}\right)=d\left(u_{1}, u_{2}\right) .
$$

That is,

$$
\sigma(r) p_{\alpha}\left(u_{1}, F u_{1}\right) \leq d\left(u_{1}, u_{2}\right)
$$

So, we have

$$
\begin{aligned}
d\left(u_{2}, u_{3}\right) & \leq D_{\alpha}\left(F u_{1}, F u_{2}\right) \\
& \leq r \max \left\{d\left(u_{1}, u_{2}\right), p_{\alpha}\left(u_{1}, F u_{1}\right), p_{\alpha}\left(u_{2}, F u_{2}\right), \frac{p_{\alpha}\left(u_{1}, F u_{2}\right)+p_{\alpha}\left(u_{2}, F u_{1}\right)}{2}\right\}
\end{aligned}
$$




$$
\begin{aligned}
& \leq r_{1} \max \left\{d\left(u_{1}, u_{2}\right), d\left(u_{1}, u_{2}\right), d\left(u_{2}, u_{3}\right), \frac{d\left(u_{1}, u_{2}\right)+p_{\alpha}\left(u_{2}, F u_{2}\right)}{2}\right\} \\
& \leq r_{1} \max \left\{d\left(u_{1}, u_{2}\right), d\left(u_{2}, u_{3}\right), \frac{d\left(u_{1}, u_{2}\right)+d\left(u_{2}, u_{3}\right)}{2}\right\} .
\end{aligned}
$$

Note that $d\left(u_{2}, u_{3}\right) \leq d\left(u_{1}, u_{2}\right)$. If not, then the above inequality gives

$$
\begin{aligned}
d\left(u_{2}, u_{3}\right) & \leq r_{1} \max \left\{d\left(u_{2}, u_{3}\right), d\left(u_{2}, u_{3}\right), \frac{d\left(u_{2}, u_{3}\right)+d\left(u_{2}, u_{3}\right)}{2}\right\} \\
& =r_{1} d\left(u_{2}, u_{3}\right)<d\left(u_{2}, u_{3}\right) \quad \text { as } r_{1}<1,
\end{aligned}
$$

a contradiction. Hence, $d\left(u_{2}, u_{3}\right) \leq r_{1} d\left(u_{1}, u_{2}\right)$. Continuing this process, we construct a sequence $\left\{u_{n}\right\}$ in $X$ such that $u_{n+1} \in\left(F u_{n}\right)_{\alpha}$ and $u_{n+2} \in\left(F u_{n+1}\right)_{\alpha}$ with

$$
d\left(u_{n+1}, u_{n+2}\right)=p_{\alpha}\left(u_{n+1}, F u_{n+1}\right) \leq D_{\alpha}\left(F u_{n}, F u_{n+1}\right) .
$$

By the given assumption, we have $\left(u_{n}, u_{n+1}\right) \in \nabla$ and $\left(u_{n+1}, u_{n+2}\right) \in \nabla$. As $\sigma(r)<1$, so

$$
\sigma(r) p_{\alpha}\left(u_{n}, F u_{n}\right) \leq p_{\alpha}\left(u_{n}, F u_{n}\right)=d\left(u_{n}, u_{n+1}\right)
$$

Therefore,

$$
\begin{aligned}
d\left(u_{n+1}, u_{n+2}\right) \leq & D_{\alpha}\left(F u_{n}, F u_{n+1}\right) \\
\leq & r \max \left\{d\left(u_{n}, u_{n+1}\right), p_{\alpha}\left(u_{n}, F u_{n}\right), p_{\alpha}\left(u_{n+1}, F u_{n+1}\right),\right. \\
& \left.\frac{p_{\alpha}\left(u_{n}, F u_{n+1}\right)+p_{\alpha}\left(u_{n+1}, F u_{n}\right)}{2}\right\} \\
\leq & r_{1} \max \left\{d\left(u_{n}, u_{n+1}\right), d\left(u_{n}, u_{n+1}\right), d\left(u_{n+1}, u_{n+2}\right),\right. \\
& \left.\frac{d\left(u_{n}, u_{n+1}\right)+p_{\alpha}\left(u_{n+1}, F u_{n+1}\right)}{2}\right\} \\
\leq & r_{1} \max \left\{d\left(u_{n}, u_{n+1}\right), d\left(u_{n+1}, u_{n+2}\right), \frac{d\left(u_{n}, u_{n+1}\right)+d\left(u_{n+1}, u_{n+2}\right)}{2}\right\} .
\end{aligned}
$$

We claim that $d\left(u_{n+1}, u_{n+2}\right) \leq d\left(u_{n}, u_{n+1}\right)$. If not, then by the above inequality, we obtain

$$
\begin{aligned}
d\left(u_{n+1}, u_{n+2}\right) & \leq r_{1} \max \left\{d\left(u_{n+1}, u_{n+2}\right), d\left(u_{n+1}, u_{n+2}\right), \frac{d\left(u_{n+1}, u_{n+2}\right)+d\left(u_{n+1}, u_{n+2}\right)}{2}\right\} \\
& \leq r_{1} d\left(u_{n+1}, u_{n+2}\right)<d\left(u_{n+1}, u_{n+2}\right),
\end{aligned}
$$

a contradiction as $r_{1}<1$. So, we have

$$
d\left(u_{n+1}, u_{n+2}\right) \leq r_{1} d\left(u_{n}, u_{n+1}\right) \leq \cdots \leq\left(r_{1}\right)^{n} d\left(u_{1}, u_{2}\right)
$$

and

$$
\sum_{n=1}^{\infty} d\left(u_{n+1}, u_{n+2}\right) \leq \sum_{n=1}^{\infty}\left(r_{1}\right)^{n} d\left(u_{1}, u_{2}\right)<\infty .
$$


Hence, $\left\{u_{n}\right\}$ is a Cauchy sequence in $X$. Since $X$ is complete, there is some point $z \in X$ such that $\lim _{n \rightarrow \infty} u_{n}=z$. As $\left(u_{n}, u_{n+1}\right) \in \nabla$ for all $n$, then by the assumption, $\left(u_{n}, z\right) \in \nabla$. Now, we show that for every pair $(x, z) \in \nabla$ with $x \neq z$, the following inequality holds:

$$
p_{\alpha}(z, T x) \leq r \max \left\{d(z, x), p_{\alpha}(x, F x)\right\}
$$

As $\lim _{n \rightarrow \infty} u_{n}=z$, there exists a positive integer $n_{0} \in N$ such that for all $n \geq n_{0}$, we have

$$
d\left(z, u_{n}\right) \leq \frac{1}{3} d(z, x)
$$

Now, for all $n \geq n_{0}$,

$$
\begin{aligned}
\sigma(r) p_{\alpha}\left(u_{n}, F u_{n}\right) & \leq p_{\alpha}\left(u_{n}, F u_{n}\right) \\
& \leq d\left(u_{n}, z\right)+p_{\alpha}\left(z, F u_{n}\right) \leq d\left(u_{n}, z\right)+d\left(z, u_{n+1}\right) \\
& \leq \frac{1}{3} d(z, x)+\frac{1}{3} d(z, x) \leq \frac{2}{3} d(z, x) \\
& =d(z, x)-\frac{1}{3} d(z, x) \\
& \leq d(z, x)-d\left(u_{n}, z\right) \leq d\left(u_{n}, x\right)
\end{aligned}
$$

implies that

$$
\begin{aligned}
p_{\alpha}\left(u_{n+1}, F x\right) & \leq D_{\alpha}\left(F u_{n}, F x\right) \\
& \leq r \max \left\{d\left(u_{n}, x\right), p_{\alpha}\left(u_{n}, F u_{n}\right), p_{\alpha}(x, F x), \frac{p_{\alpha}\left(u_{n}, F x\right)+p_{\alpha}\left(x, F u_{n}\right)}{2}\right\} \\
& \leq r \max \left\{d\left(u_{n}, x\right), d\left(u_{n}, u_{n+1}\right), p_{\alpha}(x, F x), \frac{p_{\alpha}\left(u_{n}, F x\right)+d\left(x, u_{n+1}\right)}{2}\right\},
\end{aligned}
$$

which on taking limit as $n \rightarrow \infty$ gives

$$
p_{\alpha}(z, F x) \leq r \max \left\{d(z, x), p_{\alpha}(x, F x), \frac{p_{\alpha}(z, F x)+d(x, z)}{2}\right\} .
$$

If

$$
\max \left\{d(z, x), p_{\alpha}(x, F x), \frac{p_{\alpha}(z, F x)+d(x, z)}{2}\right\}=\frac{p_{\alpha}(z, F x)+d(x, z)}{2},
$$

then

$$
\begin{aligned}
& p_{\alpha}(z, F x) \leq r \frac{p_{\alpha}(z, F x)+d(x, z)}{2} \leq \frac{r}{2} p_{\alpha}(z, F x)+\frac{r}{2} d(x, z), \\
& p_{\alpha}(z, F x) \leq \frac{r}{2-r} d(x, z) \leq \frac{r}{2-r} d(x, z) \leq r d(x, z) .
\end{aligned}
$$

Hence,

$$
p_{\alpha}(z, F x) \leq r \max \left\{d(z, x), p_{\alpha}(x, F x)\right\}
$$


Now, we show that $z_{\alpha} \subset F z$ for each $\alpha \in[0,1]$. First, consider the case $0 \leq r<1 / 2$. Assume on the contrary that $z_{\alpha} \nsubseteq F z$, that is, $z \notin(F z)_{\alpha}$. Let $a \in(F z)_{\alpha}$, as $(F z)_{\alpha}$ is nonempty and compact, so for each $\alpha \in[0,1]$, we have

$$
2 r d(a, z)<p_{\alpha}(z, F z)
$$

Now, $a \in(F z)_{\alpha}$ implies $(a, z) \in \nabla$ and $a \neq z$. From (5) we have

$$
p_{\alpha}(z, F a) \leq r \max \left\{d(z, a), p_{\alpha}(a, F a)\right\} .
$$

Now,

$$
\sigma(r) p_{\alpha}(z, F z) \leq p_{\alpha}(z, F z)=d(z, a)
$$

implies that

$$
\begin{aligned}
D_{\alpha}(F z, F a) & \leq r \max \left\{d(z, a), p_{\alpha}(z, F z), p_{\alpha}(a, F a), \frac{p_{\alpha}(z, F a)+p_{\alpha}(a, F z)}{2}\right\} \\
& \leq r \max \left\{d(z, a), p_{\alpha}(z, F z), p_{\alpha}(a, F a), \frac{p_{\alpha}(z, F a)}{2}\right\} \\
& \leq r \max \left\{d(z, a), p_{\alpha}(z, F z), p_{\alpha}(a, F a), \frac{d(z, a)+p_{\alpha}(a, F a)}{2}\right\} \\
& \leq r \max \left\{d(z, a), p_{\alpha}(z, F z), p_{\alpha}(a, F a)\right\} \\
& \leq r \max \left\{d(z, a), p_{\alpha}(a, F a)\right\} .
\end{aligned}
$$

Hence,

$$
D_{\alpha}(F z, F a) \leq r \max \left\{d(z, a), p_{\alpha}(a, F a)\right\},
$$

which further implies that

$$
p_{\alpha}(a, F a) \leq r \max \left\{d(z, a), p_{\alpha}(a, F a)\right\} \quad \text { as } p_{\alpha}(a, F a) \leq D_{\alpha}(F z, F a) .
$$

We claim that $p_{\alpha}(a, F a) \leq d(z, a)$. If not, then the above inequality becomes

$$
p_{\alpha}(a, F a) \leq r p_{\alpha}(a, F a)<p_{\alpha}(a, F a) \quad \text { as } r<1,
$$

a contradiction, so we deduce that $p_{\alpha}(a, F a) \leq r d(z, a)$. From inequality (7), we have

$$
p_{\alpha}(z, F a) \leq r d(z, a)
$$

Therefore,

$$
\begin{aligned}
p_{\alpha}(z, F z) & \leq p_{\alpha}(z, F a)+D_{\alpha}(F z, F a) \\
& \leq r d(z, a)+r \max \left\{d(z, a), p_{\alpha}(a, F a)\right\} \\
& \leq r d(z, a)+r d(z, a) \leq 2 r d(z, a)<p_{\alpha}(z, F z),
\end{aligned}
$$

a contradiction. Hence, $z_{\alpha} \subset F z$. 
Now, when $1 / 2 \leq r<1$, we first prove that

$$
D_{\alpha}(F x, F z) \leq r \max \left\{d(x, z), p_{\alpha}(x, F x), p_{\alpha}(z, F z), \frac{p_{\alpha}(x, F z)+p_{\alpha}(z, F x)}{2}\right\}
$$

for all $(x, z) \in \nabla$. If $x=z$, then (8) holds trivially. So, assume that $x \neq z$. For every $n \in N$, one may find a sequence $y_{n} \in(F x)_{\alpha}$ such that

$$
d\left(z, y_{n}\right) \leq p_{\alpha}(z, F x)+\frac{1}{n} d(x, z)
$$

As $y_{n} \in(F x)_{\alpha}$, this implies $\left(y_{n}, x\right) \in \nabla$. Using (7) we have

$$
\begin{aligned}
p_{\alpha}(x, F x) & \leq d\left(x, y_{n}\right) \\
& \leq d(x, z)+d\left(z, y_{n}\right) \\
& \leq d(x, z)+p_{\alpha}(z, F x)+\frac{1}{n} d(x, z) \\
& \leq d(x, z)+r \max \left\{d(z, x), p_{\alpha}(x, F x)\right\}+\frac{1}{n} d(x, z)
\end{aligned}
$$

for all $n \in N$. If $d(x, z) \geq p_{\alpha}(x, F x)$, then

$$
\begin{aligned}
p_{\alpha}(x, F x) & \leq d(x, z)+r d(z, x)+\frac{1}{n} d(x, z) \\
& \leq d(x, z)+r d(z, x)+\frac{1}{n} d(x, z) \leq\left(1+r+\frac{1}{n}\right) d(x, z) .
\end{aligned}
$$

This implies that

$$
\frac{1}{(1+r)} p_{\alpha}(x, F x) \leq\left(1+\frac{1}{(1+r) n}\right) d(x, z)
$$

Hence, for $\frac{1}{2} \leq r<1$, we obtain

$$
\begin{aligned}
\sigma(r) p_{\alpha}(x, F x) & =(1-r) p_{\alpha}(x, F x) \\
& \leq \frac{1}{(1+r)} p_{\alpha}(x, F x) \leq\left(1+\frac{1}{(1+r) n}\right) d(x, z) .
\end{aligned}
$$

On taking the limit as $n \rightarrow \infty$, we have

$$
\sigma(r) p_{\alpha}(x, F x) \leq d(x, z)
$$

If $d(x, z) \leq p_{\alpha}(x, F x)$, then

$$
\begin{aligned}
& p_{\alpha}(x, F x) \leq d(x, z)+r p_{\alpha}(x, F x)+\frac{1}{n} d(x, z), \\
& p_{\alpha}(x, F x)-r p_{\alpha}(x, F x) \leq d(x, z)+\frac{1}{n} d(x, z), \\
& (1-r) p_{\alpha}(x, F x) \leq d(x, z)+\frac{1}{n} d(x, z)
\end{aligned}
$$


On taking the limit as $n \rightarrow \infty$, we have

$$
\sigma(r) p_{\alpha}(x, F x) \leq d(x, z)
$$

By the given assumption, we have

$$
D_{\alpha}(F x, F z) \leq r \max \left\{d(x, z), p_{\alpha}(x, F x), p_{\alpha}(z, F z), \frac{p_{\alpha}(x, F z)+p_{\alpha}(z, F x)}{2}\right\} .
$$

Thus, for any $x \neq z$, (8) holds true. Put $x=u_{n}$ in the above inequality to obtain

$$
\begin{aligned}
p_{\alpha}(z, F z) & \leq \lim _{n \rightarrow \infty} p_{\alpha}\left(u_{n+1}, F z\right) \leq \lim _{n \rightarrow \infty} D_{\alpha}\left(F u_{n}, F z\right) \\
& \leq \lim _{n \rightarrow \infty} r \max \left\{d\left(u_{n}, z\right), d\left(u_{n}, u_{n+1}\right), p_{\alpha}(z, F z), \frac{p_{\alpha}\left(u_{n}, F z\right)+p_{\alpha}\left(z, F u_{n}\right)}{2}\right\} \\
& =r p_{\alpha}(z, F z)
\end{aligned}
$$

as $r<1$, we get $p_{\alpha}(z, F z)=0$. Hence by Lemma $2, z_{\alpha} \subset F z$.

Corollary 5 Let $(X, d, \preceq)$ be a complete ordered metric space. If an ordered fuzzy mapping $F: X \rightarrow W_{\alpha}(X)$ satisfies

$$
\sigma(r) p_{\alpha}(x, F x) \leq d(x, y) \quad \text { implies } \quad D_{\alpha}(F x, F y) \leq r M_{\alpha}(F)
$$

for all $(x, y) \in \nabla$, where

$$
M_{\alpha}(F)=\max \left\{d(x, y), p_{\alpha}(x, F x), p_{\alpha}(y, F y)\right\} .
$$

Then there exists a point $x \in X$ such that $x_{\alpha} \subset F x$ provided that $X$ satisfies the order sequential limit property.

Corollary 6 Let $(X, d, \preceq)$ be a complete ordered metric space. If an ordered fuzzy mapping $F: X \rightarrow W_{\alpha}(X)$ satisfies

$$
\sigma(r) p_{\alpha}(x, F x) \leq d(x, y) \quad \text { implies } \quad D_{\alpha}(F x, F y) \leq \lambda M_{\alpha}(F)
$$

for all $(x, y) \in \nabla$, where

$$
\left.M_{\alpha}(F)=d(x, y)+p_{\alpha}(x, F x)+p_{\alpha}(y, F y)\right\}
$$

and $\lambda \in\left[0, \frac{1}{3}\right), r=3 \lambda$. Then there exists a point $x \in X$ such that $x_{\alpha} \subset F x$ provided that $X$ satisfies the order sequential limit property.

\section{An application}

Let $F: X \rightarrow W_{\alpha}(X)$ and $g: X \rightarrow X$. A pair $\{F, g\}$ is said to be an ordered fuzzy hybrid pair if the following conditions are satisfied:

(c) $g y \in F(x)_{\alpha}$ implies that $(y, x) \in \nabla$.

(d) $(x, y) \in \nabla$ gives $(u, v) \in \nabla$ whenever $g u \in(F x)_{\alpha}$ and $g v \in(F y)_{\alpha}$.

(e) $(g x, g y) \in \nabla$ whenever $(x, y) \in \nabla$ for all $x, y \in X$. 
Theorem 7 Let $(X, d, \preceq)$ be a complete ordered metric space. If an ordered fuzzy hybrid pair $\{F, g\}$ satisfies

$$
\sigma(r) p_{\alpha}(g x, F x) \leq d(g x, g y) \quad \text { implies } \quad D_{\alpha}(F x, F y) \leq r M_{\alpha}(F, g)
$$

for all $(x, y) \in \nabla$, where

$$
M_{\alpha}(F, g)=\max \left\{d(g x, g y), p_{\alpha}(g x, F x), p_{\alpha}(g y, F y), \frac{p_{\alpha}(g x, F y)+p_{\alpha}(g y, F x)}{2}\right\} .
$$

Then $C_{\alpha}(F, g) \neq \phi$ provided that $X$ satisfies the order sequential limit property and $(F(X))_{\alpha} \subseteq g(X)$ for each $\alpha$. Moreover, $F$ and $g$ have a common fixed fuzzy point if any of the following conditions holds:

(f) $F$ and $g$ are $w$-fuzzy compatible, $\lim _{n \rightarrow \infty} g^{n} x=u$ and $\lim _{n \rightarrow \infty} g^{n} y=v$ for some $x \in C_{\alpha}(F, g), u \in X$ and $g$ is continuous at $u$.

(g) $g$ is $F$-fuzzy weakly commuting for some $x \in C_{\alpha}(g, F)$ and is a fixed point of $g$, that is, $g^{2} x=g x$.

(h) $g$ is continuous at $x$ for some $x \in C_{\alpha}(g, F)$ and for some $u \in X$ such that $\lim _{n \rightarrow \infty} g^{n} u=x$.

Proof By Lemma 1, there exists $E \subseteq X$ such that $g: E \rightarrow X$ is one-to-one and $g(E)=g(X)$. Define a mapping $\mathcal{A}: g(E) \rightarrow W_{\alpha}(X)$ by

$$
\mathcal{A} g x=F x \quad \text { for all } g x \in g(E) \text {. }
$$

As $g$ is one-to-one on $E, \mathcal{A}$ is well defined. Also,

$$
\sigma(r) p_{\alpha}(g x, F x) \leq d(g x, g y) \quad \text { implies } \quad D_{\alpha}(F x, F y) \leq r M_{\alpha}(F, g)
$$

for all $(x, y) \in \nabla$. Therefore,

$$
\sigma(r) p_{\alpha}(g x, \mathcal{A} g x) \leq d(g x, g y) \quad \text { implies } \quad D_{\alpha}(\mathcal{A} g x, \mathcal{A} g y) \leq r M_{\alpha}(F, g)
$$

for all $(g x, g y) \in \nabla$. Hence, $\mathcal{A}$ satisfies (2) and all the conditions of Theorem 4. Using Theorem 4 with a mapping $\mathcal{A}$, it follows that $\mathcal{A}$ has a fixed fuzzy point $u \in g(E)$. Now, it is left to prove that $F$ and $g$ have a coincidence fuzzy point. Since $\mathcal{A}$ has a fixed fuzzy point $u_{\alpha} \subset \mathcal{A} u$, we get $u \in(\mathcal{A} u)_{\alpha}$. As $(F(X))_{\alpha} \subseteq g(X)$, so there exists $u_{1} \in X$ such that $g u_{1}=u$, thus it follows that $g u_{1} \in\left(\mathcal{A} g u_{1}\right)_{\alpha}=\left(F u_{1}\right)_{\alpha}$. This implies that $u_{1} \in X$ is a coincidence fuzzy point of $F$ and $g$. Hence, $C_{\alpha}(F, g) \neq \phi$. Suppose now that (f) holds. Then for some $x_{\alpha} \in C_{\alpha}(F, g)$, we have $\lim _{n \rightarrow \infty} g^{n} x=u$, where $u \in X$. Thus $\left(g^{n-1} x, u\right) \in \nabla$. Since $g$ is continuous at $u$, we have that $u$ is a fixed point of $g$. As $F$ and $g$ are $w$-fuzzy compatible, and $\left(g^{n} x\right)_{\alpha} \in C_{\alpha}(F, g)$ for all $n \geq 1$. That is, $g^{n} x \in F\left(g^{n-1} x\right)_{\alpha}$ for all $n \geq 1$. Now,

$$
\begin{aligned}
\sigma(r) p_{\alpha}\left(g^{n} x, F g^{n-1} x\right) & \leq p_{\alpha}\left(g^{n} x, F g^{n-1} x\right)=0 \\
& \leq d\left(g g^{n-1} x, g u\right)
\end{aligned}
$$


implies that

$$
\begin{aligned}
p_{\alpha}(g u, F u) \leq & p_{\alpha}\left(g u, g^{n} x\right)+p_{\alpha}\left(g^{n} x, F u\right) \\
\leq & p_{\alpha}\left(g u, g^{n} x\right)+D_{\alpha}\left(F\left(g^{n-1} x\right), F u\right) \\
\leq & p_{\alpha}\left(g u, g^{n} x\right)+r \max \left\{d\left(g g^{n-1} x, g u\right), p_{\alpha}\left(g^{n} x, F g^{n-1} x\right), p_{\alpha}(g u, F u),\right. \\
& \left.\frac{p_{\alpha}\left(g u, F g^{n-1} x\right)+p_{\alpha}\left(g^{n} x, F u\right)}{2}\right\} .
\end{aligned}
$$

On taking limit as $n \rightarrow \infty$, we get $p_{\alpha}(g u, F u) \leq r p_{\alpha}(g u, F u)$ and therefore $p_{\alpha}(g u, F u)=0$. By Lemma 2 we obtain $g u \in(F u)_{\alpha}$. Consequently, $u=g u \in(F u)_{\alpha}$. Hence, $u_{\alpha}$ is a common fixed fuzzy point of $F$ and $g$. Suppose now that (g) holds. If for some $x_{\alpha} \in C_{\alpha}(F, g), g$ is $F$-fuzzy weakly commuting and $g^{2} x=g x$, then $g x=g^{2} x \in(F g x)_{\alpha}$. Hence, $(g x)_{\alpha}$ is a common fixed fuzzy point of $F$ and $g$. Suppose now that (h) holds and assume that for some $x_{\alpha} \in C_{\alpha}(F, g)$ and for some $u \in X, \lim _{n \rightarrow \infty} g^{n} u=x$ and $\lim _{n \rightarrow \infty} g^{n} v=y$. By the continuity of $g$ at $x$ and $y$, we get $x=g x \in(F x)_{\alpha}$. The result follows.

Example 1 Let $X=[0,1]$ be endowed with the usual metric. Let $\alpha \in\left(0, \frac{1}{3}\right)$ and $r=\frac{1}{2}$, then $\sigma(r)=\frac{1}{2}$. Define a fuzzy mapping $F$ from $X$ into $W_{\alpha}(X)$ as

$$
(F 0)(x)=\left\{\begin{array}{ll}
1 & \text { if } x=0, \\
\alpha & \text { if } x \in\left(0, \frac{1}{3}\right], \\
\frac{\alpha}{3} & \text { if } x \in\left(\frac{1}{3}, 1\right]
\end{array} \quad \text { and } \quad(F 1)(x)= \begin{cases}1 & \text { if } x=0, \\
3 \alpha & \text { if } x \in\left(0, \frac{1}{3}\right] \\
\frac{\alpha}{3} & \text { if } x \in\left(\frac{1}{3}, 1\right]\end{cases}\right.
$$

and for $z \in(0,1)$,

$$
(F z)(x)= \begin{cases}1 & \text { if } x=0 \\ \alpha & \text { if } x \in\left(0, \frac{1}{3}\right] \\ 0 & \text { if } x \in\left(\frac{1}{3}, 1\right]\end{cases}
$$

Define a self-map $g: X \rightarrow X$ by $g(x)=x^{2}$. Then

$$
\begin{aligned}
& (F 0)_{1}=(F 1)_{1}=(F z)_{1}=\{0\}, \quad(F 0)_{\alpha}=(F 1)_{\alpha}=(F z)_{\alpha}=\left[0, \frac{1}{3}\right], \\
& (F 0)_{\frac{\alpha}{3}}=(F 1)_{\frac{\alpha}{3}}=[0,1] \text { and } \quad(F z)_{\frac{\alpha}{3}}=\left[0, \frac{1}{3}\right] .
\end{aligned}
$$

Note that for all $x, y \in X$, we have

$$
D_{1}(F x, F y)=H\left((F x)_{1},(F y)_{1}\right)=D_{\alpha}(F x, F y)=H\left((F x)_{\alpha},(F y)_{\alpha}\right)=0 .
$$

Also, for all $x, y \in\{0,1\}$, we have

$$
D_{\frac{\alpha}{3}}(F x, F y)=H\left((F x)_{\frac{\alpha}{3}},(F y)_{\frac{\alpha}{3}}\right)=0 .
$$


And

$$
D_{\frac{\alpha}{3}}(F x, F y)=H\left((F x)_{\frac{\alpha}{3}},(F y)_{\frac{\alpha}{3}}\right)=0 \quad \text { for all } x, y \in(0,1) .
$$

If $x \in\{0,1\}$ and $y \in(0,1)$, then $D_{\frac{\alpha}{3}}(F x, F y)=H\left((F x)_{\frac{\alpha}{3}},(F y)_{\frac{\alpha}{3}}\right)=\frac{2}{3}$. So, for all $x, y \in X$, with $\sigma(r) p_{\alpha}(x, F x) \leq d(x, y)$, we have $D_{\alpha}(F x, F y)=0$. Hence, for all $x, y \in X$,

$$
D_{\alpha}(F x, F y) \leq r M_{\alpha}(F) \quad \text { and } \quad D_{\alpha}(F x, F y) \leq r M_{\alpha}(F, g)
$$

hold true, where

$$
M_{\alpha}(F)=\max \left\{d(x, y), p_{\alpha}(x, F x), p_{\alpha}(y, F y), \frac{p_{\alpha}(x, F y)+p_{\alpha}(y, F x)}{2}\right\}
$$

and

$$
M_{\alpha}(F, g)=\max \left\{d(g x, g y), p_{\alpha}(g x, F x), p_{\alpha}(g y, F y), \frac{p_{\alpha}(g x, F y)+p_{\alpha}(g y, F x)}{2}\right\} .
$$

Hence, all the conditions of Theorem 7 are satisfied. Moreover, for each $x \in\left[0, \frac{1}{3}\right]$, we have $x_{\alpha} \subset F(x)$ and $(g x)_{\alpha} \subset F(x)$. For $\alpha=1$, we have $\{0\}=\{g 0\} \subset(F 0)_{1}$.

\section{Conclusion}

The Banach contraction principle has become a classical tool to show the existence of solutions of functional equations in nonlinear analysis (see for details [23-26]). Suzuki-type fixed point theorems $[10,14]$ are the generalizations of the Banach contraction principle that characterize metric completeness of underlying spaces. Fuzzy sets and mappings play important roles in the process of fuzzification of systems. Suzuki-type fixed point theorems for fuzzy mappings obtained in this article can further be used in the process of finding the solutions of functional equations involving fuzzy mappings in fuzzy systems. In the main result, we not only extended the mapping to a fuzzy mapping, but also the underlying metric space has been replaced with ordered metric spaces. In this article, we defined coincidence fuzzy points and common fixed fuzzy points of the hybrid pair of a single-valued self-mapping and a fuzzy mapping and applied our main result to obtain the existence of coincidence fuzzy points and common fixed fuzzy points of the hybrid pair.

Competing interests

The authors declare that they have no competing interests.

Authors' contributions

All authors read and approved the final manuscript.

Author details

'Department of Mathematics, School of Science and Engineering, Lahore University of Management Sciences, Lahore, 54792, Pakistan. ${ }^{2}$ Department of Mathematics and Applied Mathematics, University of Pretoria, Hatfield, Pretoria, South Africa.

Acknowledgements

The authors are thankful to the referees for their critical remarks which helped to improve the presentation of this paper. 


\section{References}

1. Heilpern, S: Fuzzy mappings and fuzzy fixed point theorems. J. Math. Anal. Appl. 83, 566-569 (1981)

2. Sen, CS: Fixed degree for fuzzy mappings and a generalization of Ky Fan's theorem. Fuzzy Sets Syst. 24, 103-112 (1987)

3. Estruch, VD, Vidal, A: A note on fixed fuzzy points for fuzzy mappings. Rend. Ist. Mat. Univ. Trieste 32, 39-45 (2001)

4. Haghi, RH, Rezapour, S, Shahzad, N: Some fixed point generalizations are not real generalizations. Nonlinear Anal. 74, 1799-1803 (2011)

5. Lee, BS, Cho, SJ: A fixed point theorem for contractive type fuzzy mappings. Fuzzy Sets Syst. 61, 309-312 (1994)

6. Zadeh, LA: Fuzzy sets. Inf. Control 8, 103-112 (1965)

7. Nadler, SB Jr.: Multivalued contraction mappings. Pac. J. Math. 30, 475-488 (1969)

8. Sedghi, S, Shobe, N, Altun, I: A fixed fuzzy point for fuzzy mappings in complete metric spaces. Math. Commun. 13, 289-294 (2008)

9. Turkoglu, D, Rhoades, BE: A fixed fuzzy point for fuzzy mapping in complete metric spaces. Math. Commun. 10 115-121 (2005)

10. Suzuki, T: A generalized Banach contraction principle that characterizes metric completeness. Proc. Am. Math. Soc $136,1861-1869$ (2008)

11. Altun, I, Erduran, A: A Suzuki type fixed-point theorem. Int. J. Math. Math. Sci. 2011, Article ID 736063 (2011). doi:10.1155/2011/736063

12. Ćirić, L, Abbas, M, Rajović, M, Ali, B: Suzuki type fixed point theorems for generalized multi-valued mappings on a set endowed with two b-metrics. Appl. Math. Comput. 219, 1712-1723 (2012)

13. Singh, SL, Mishra, SN: Coincidence theorems for certain classes of hybrid contractions. Fixed Point Theory Appl. 2010, Article ID 898109 (2010)

14. Dorić, D, Lazović, R: Some Suzuki type fixed point theorems for generalized multivalued mappings and applications. Fixed Point Theory Appl. 2011, 40 (2011)

15. Ran, ACM, Reurings, MCB: A fixed point theorem in partially ordered sets and some application to matrix equations. Proc. Am. Math. Soc. 132, 1435-1443 (2004)

16. Abbas, M, Khamsi, MA, Khan, AR: Common fixed point and invariant approximation in hyperbolic ordered metric spaces. Fixed Point Theory Appl. 2011, 25 (2011). doi:10.1186/1687-1812-2011-25

17. Amini-Harandi, A, Emami, H: A fixed point theorem for contraction type maps in partially ordered metric spaces and application to ordinary differential equations. Nonlinear Anal. 72, 2238-2242 (2010)

18. Ćirić, L, Abbas, M, Saadati, R, Hussain, N: Common fixed points of almost generalized contractive mappings in ordered metric spaces. Appl. Math. Comput. 217, 5784-5789 (2011)

19. Harjani, J, Sadarangani, K: Fixed point theorems for weakly contractive mappings in partially ordered sets. Nonlinear Anal. 71, 3403-3410 (2009)

20. Kadelburg, Z, Pavlović, M, Radenović, S: Common fixed point theorems for ordered contractions and quasicontractions in ordered cone metric spaces. Comput. Math. Appl. 59, 3148-3159 (2010)

21. Nieto, JJ, Lopez, RR: Contractive mapping theorems in partially ordered sets and applications to ordinary differential equations. Order 22, 223-239 (2005)

22. Samet, B: Coupled fixed point theorems for a generalized Meir-Keeler contraction in partially ordered metric spaces. Nonlinear Anal. 72, 4508-4517 (2010)

23. Baskaran, R, Subrahmanyam, PV: A note on the solution of a class of functional equations. Appl. Anal. 22, 235-241 (1986)

24. Bellman, R: Methods of Nonlinear Analysis. Vol. II. Mathematics in Science and Engineering, vol. 61. Academic Press, New York (1973)

25. Bellman, R, Lee, ES: Functional equations in dynamic programming. Aequ. Math. 17, 1-18 (1978)

26. Bhakta, PC, Mitra, S: Some existence theorems for functional equations arising in dynamic programming. J. Math. Anal. Appl. 98, 348-362 (1984)

\section{Submit your manuscript to a SpringerOpen ${ }^{0}$ journal and benefit from:}

- Convenient online submission

- Rigorous peer review

- Immediate publication on acceptance

- Open access: articles freely available online

- High visibility within the field

- Retaining the copyright to your article

Submit your next manuscript at $>$ springeropen.com 